\title{
A randomized double-blind controlled study on the efficacy and safety of Sangdantongluo granule in the treatment of post-stroke spasticity
}

lexie ( $\sim 1289398706 @ q q . c o m)$

Hunan Academy of Chinese Medicine Affiliated Hospital: Hunan University of Chinese Medicine Integrated Chinese Medicine Affiliated Hospital https://orcid.org/0000-0002-2452-1383

\section{Yao Xie}

Hunan Academy of Chinese Medicine Affiliated Hospital: Hunan University of Chinese Medicine Integrated Chinese Medicine Affiliated Hospital

\section{Guo Mao}

Hunan Academy of Chinese Medicine Affiliated Hospital: Hunan University of Chinese Medicine Integrated Chinese Medicine Affiliated Hospital

\section{Junlin Jiang}

Hunan Academy of Chinese Medicine Affiliated Hospital: Hunan University of Chinese Medicine Integrated Chinese Medicine Affiliated Hospital

\section{Ting Yao}

Hunan Academy of Chinese Medicine Affiliated Hospital: Hunan University of Chinese Medicine Integrated Chinese Medicine Affiliated Hospital

\section{Rui Fang}

Hunan University of Chinese Medicine

\section{Zenghui Yue}

Hunan University of Chinese Medicine

\section{Yingchen Li}

Hunan Academy of Chinese Medicine Affiliated Hospital: Hunan University of Chinese Medicine Integrated Chinese Medicine Affiliated Hospital

\section{Dahua Wu}

Hunan Academy of Chinese Medicine Affiliated Hospital: Hunan University of Chinese Medicine Integrated Chinese Medicine Affiliated Hospital https://orcid.org/0000-0002-0943-0037

\section{Research Article}

Keywords: Traditional Chinese medicine, Sangdantongluo granule, Stroke, Spasticity 
DOI: https://doi.org/10.21203/rs.3.rs-679035/v1

License: (c) (1) This work is licensed under a Creative Commons Attribution 4.0 International License. Read Full License 


\section{Trials structured Study Protocol template}

Trials guidance: the numbers in curly brackets (e.g. $\{5 a\})$ are SPIRIT item identifiers. Please do not remove the numbers in curly brackets, or any heading that contains them. The item identifiers are slightly out of sequence to make the document flow more easily but it is important that they remain in the document to allow electronic searches by SPIRIT item number.

NB: All text in green can be removed once you have finished creating your Study Protocol. Text in black is mandatory. Please also read the submission guidelines for Study Protocols prior to submitting to Trials: https://trialsjournal.biomedcentral.com/submission-guidelines/preparing-your-manuscript/study-protocoll

\section{Title}

A randomized double-blind controlled study on the efficacy and safety of Sangdantongluo granule in the treatment of post-stroke spasticity

\section{Names protocol contributors}

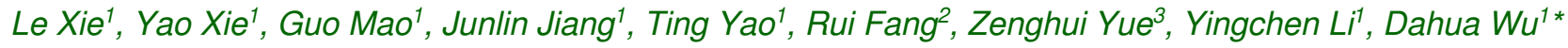

\section{Abstract}

Trials guidance: The Abstract should not exceed 350 words. Please minimize the use of abbreviations and do not cite references in the abstract. The abstract must include the following separate sections:

- Background: Stroke is the first leading cause of mortality and disability worldwide, and post-stroke spasticity (PSS) is the common complication of stroke. Sangdantongluo Granule, a modern patent Traditional Chinese medicine (TCM), is widely used in clinical practice to treat PSS. Whereas, there is limited evidence of effectiveness for Sangdantongluo Granule to treat PSS. This study will evaluate the clinical efficacy and safety of Sangdantongluo granule in the treatment of PSS.

- Methods: This multicenter, randomized, double-blind and placebo-controlled study will recruit 132 participants in China who develops PSS 15 days to 90 days after stroke. Participants will be randomly assigned in an equal ratio to receive either Sangdantongluo granule or placebo for 2 months twice a day orally. The primary measure is the Modified Ashworth Scale (MAS), Secondary outcome measures include Compopsite Spasticity Scale (CSS), Simplified Fugl-Meyer Motor Scale (S-FM), National Institute of Health stroke scale (NIHSS), Modified Rankin Scale (mRS), Modified Barther Index (MBI), and Surface electromyography. Adverse events will be supervised throughout the trial.

- Discussion: The results of this study will present whether Sangdantongluo granule is clinical effective and safe for managing PSS.

- Trial registration: ClinicalTrials.gov ChiCTR2100044544. Registered on 23 March 2021. 


\section{Keywords}

Traditional Chinese medicine, Sangdantongluo granule, Stroke, Spasticity

\section{Administrative information}

Trials guidance: please include this text in your protocol just above the Administrative information table:

Note: the numbers in curly brackets in this protocol refer to SPIRIT checklist item numbers. The order of the items has been modified to group similar items (see http://www.equator-network.org/reportingguidelines/spirit-2013-statement-defining-standard-protocol-items-for-clinical-trials/).

\begin{tabular}{|c|c|}
\hline Title $\{1\}$ & $\begin{array}{l}\text { A randomized double-blind controlled study on the efficacy and safety } \\
\text { of Sangdantongluo granule in the treatment of post-stroke spasticity. }\end{array}$ \\
\hline Trial registration $\{2 \mathrm{a}$ and $2 \mathrm{~b}\}$. & $\begin{array}{l}\text { ClinicalTrials.gov ChiCTR2100044544. Registered on } 23 \text { March } \\
\text { 2021. }\end{array}$ \\
\hline Protocol version $\{3\}$ & 09 Feb 2021, V 3.3 \\
\hline Funding $\{4\}$ & $\begin{array}{l}\text { This study will be supported by the Foundation of China Center for } \\
\text { Evidence Based Traditional Chinese Medicine [grant number } \\
\text { 2019XZZX-NB004] }\end{array}$ \\
\hline Author details $\{5 a\}$ & $\begin{array}{l}{ }^{1} \text { Neurology Department, Hunan Academy of Chinese Medicine } \\
\text { Affiliated Hospital, Changsha, Hunan, 410006; } \\
{ }^{2} \text { College of Integrated Traditional Chinese and Western Medicine, } \\
\text { Hunan University of Chinese Medicine, Changsha, Hunan, 410208; } \\
{ }^{3} \text { College of Acupuncture \& Moxibustion and Tui-na, Hunan University } \\
\text { of Chinese Medicine, Changsha, Hunan, } 410208 \text {, P.R. China; }\end{array}$ \\
\hline $\begin{array}{l}\text { Name and contact information for } \\
\text { the trial sponsor }\{5 b\}\end{array}$ & $\begin{array}{l}\text { National Administration of Traditional Chinese Medicine, contact } \\
\text { number: } 010-59957777 \text {. }\end{array}$ \\
\hline
\end{tabular}




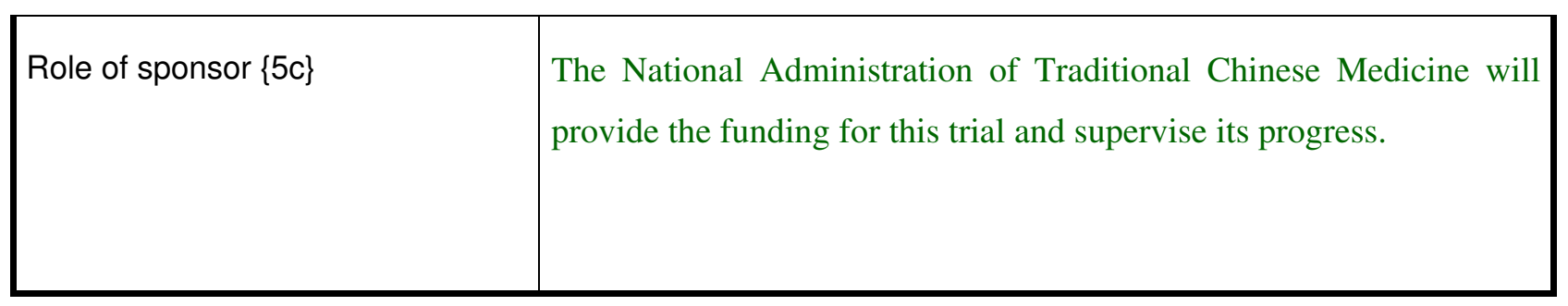

\section{Introduction}

\section{Background and rationale $\{6 \mathrm{a}\}$}

Stroke nowadays is the highest disability-adjusted life-years lost of any illness all over the China, with two million new cases per year. This situation will worsen as the consequences of population ageing and persistent high going of risk factors (smoking and hypertension) in China [1, 2]. Post-stroke spasticity (PSS) is a common symptom of upper motor neuron injury with an incidence account for $30-80 \%$, which arising from lesions to the reticulospinal or cortico-reticulospinal induced by stroke [3, 4]. Muscle tension and stiffness of PSS usually be associated with forceless and loss of dexterity, making for disordered movementcontrol, just as inability to reach, grasp, and walk [5].

In the aspect of PSS management, rehabilitation is preferred as the basic treatment, including antispasticity limb position, joint mobility training, and spastic muscle stretching slowly, but they show a relatively limited curative effect. Injection of botulinum toxin locally or oral muscle relaxants, for instance baclofen, benzodiazepines and dantrolene, have limited evidence of efficacy but with plentiful adverse effects, that restricts the widespread utilization of these medications in PSS [6, 7].

Chinese herbal products are extensively used in PSS patients in China [8]. In accordance with the theory of Traditional Chinese Medicine (TCM), yin deficiency and blood stasis are the primary syndromes in PSS. Therefore, we prescribed Sangdantongluo granule to nourish yin and promote blood circulation in the treatment of PSS, which already be widely used in clinical practice for a long time and developed into hospital preparation (Z20210481000). Sangdantongluo granule is composed of Mori Fructus, Lycii Fructus, Salviae Miltiorrhizae Radix Et Rhizoma, Lumbricus, Siegesbeckiae Herba, Paeoniae Radix Alba, Gastrodiae Rhizoma, Smilax nipponica, Cicadae Periostracum, and Crataegi Fructus. In our previous research, we confirmed that Sangdantongluo granule can relieve spasticity of hemiplegia limbs and improve motor performance in PSS patients, but with poor supporting evidence. Therefore, we prepare to conduct a multicenter, randomized, double-blind and placebo-controlled study to evaluate the clinical efficacy and safety of Sangdantongluo granule in the treatment of PSS, which may be a novel proposed approach for PSS.

\section{Objectives $\{7\}$}

The purpose of this study is to evaluate the clinical efficacy and safety of Sangdantongluo granule. Hypothesis of this study is that spasticity of hemiplegia limbs can be relieved by Sangdantongluo granule in PSS patients, compared with placebo. 


\section{Trial design $\{8\}$}

This is a multicenter, randomized, double-blind and placebo-controlled clinical trial. The aim of this trial is to to evaluate the clinical efficacy and safety of Sangdantongluo granule in the treatment of PSS.

\section{Methods: Participants, interventions and outcomes Study setting $\{9\}$}

The clinical trial will be conducted at the Hunan Academy of Chinese Medicine Affiliated Hospital, Liling Hospital of Traditional Chinese Medicine and Ningxiang Hospital of Traditional Chinese Medicine in China, with a target sample of 132 participants.

\section{Eligibility criteria $\{10\}$}

Inclusion criteria

(1) Western medicine diagnosis conforms to the diagnostic criteria of cerebral infarction;

(2) TCM syndrome meets the criteria of yin deficiency and blood stasis syndrome;

(3) Patients with limb hemiplegia and spasticity: the motor function evaluation criteria of hemiplegia in

Brunnstrom 6 stage are above stage III, modified Ashworth score (MAS) $\geq 1$, and $\leq$ grade 3 ;

(4) Age $\geq 18$ years and $\leq 80$ years old;

(5) conscious patients (GCS score $=15$ );

(6) Duration ranges from 15 days to 90 days;

(7) Patients who had not participated in other clinical trials in the last 3 months;

(8) Patients or their family members agreed and signed informed consent.

\section{Exclusion criteria}

(1) Previous motor dysfunction, such as rheumatoid arthritis, limb surgery, joint deformities, multiple sclerosis, spinal cord injury, or neuromuscular lesions that affect limb activity;

(2) Patients with a history of stroke and limb spasticity;

(3) Patients with severe hepatorenal insufficiency (defined as ALT > 2 times the normal upper limit or AST > 2 times the normal upper limit). Severe renal insufficiency is defined as $\mathrm{Cr}>1.5$ times normal upper limit);

(4) Complicated with other life-threatening diseases such as severe heart, liver, kidney and endocrine diseases with an expected survival time of less than 3 months;

(5) Inability to understand and/or follow up research procedures and/or follow-up due to mental illness, cognitive or emotional disorders;

(6) Allergic to Sangdantongluo granule;

(7) Pregnant or lactating women.

(8) Ischemic stroke caused by other non-arteriosclerosis, such as intracranial tumor, heart disease, blood disease, brain trauma and so on. 


\section{Who will take informed consent? \{26a\}}

Patients with PSS will obtain written document about the study and determine whether they agree to sign the informed consent form to take part in this study.

\section{Additional consent provisions for collection and use of participant data and biological specimens $\{26 \mathrm{~b}\}$}

Participants will be confirmed whether their clinical data can be used or shared by related organization.

\section{Interventions}

\section{Explanation for the choice of comparators $\{6 \mathrm{~b}\}$}

The Sangdantongluo granule placebo was choosed as the comparator to give evidence of the clinical efficacy and safety of Sangdantongluo granule in the treatment of PSS.

\section{Intervention description $\{11 \mathrm{a}\}$}

Eligible participants will be randomly distributed to experimental group or control group in a ration of $1: 1$. All participants will receive standard conventional treatments, under the Chinese Guidelines for the Prevention of Stroke in Patients with Stroke and Transient Ischemic Attack 2014 and Chinese Guidelines for early rehabilitation of stroke $[9,10]$. Standard conventional treatments in the trial as follows:

(1) Risk factor control: blood pressure, blood glucose and blood lipid management, smoking cessation, etc;

(2) Antiplatelet agents: aspirin (100 mg, once per day) or clopidogrel (75 mg, once per day);

(3) Conventional rehabilitation: anti-spasticity limb position, joint mobility training, and spastic muscle stretching slowly.

The experimental group will be treated by Sangdantongluo granule for 2 months twice a day orally on the basis of standard conventional treatments. While the control group will be treated by placebo granule, which be made from starch with the similar appearance, color, and odor also for 2 months twice a day orally.

\section{Criteria for discontinuing or modifying allocated interventions $\{11 \mathrm{~b}\}$}

The criteria for discontinuation are as follows:

(1) Condition aggravation during the study;

(2) Participants who develop serious complications;

(3) Participants with poor compliance, such as the use of experimental drug can not reach $80 \%$;

(4) Cases of breaking the blind or urgently unblinding;

(5) Participants withdraw from the trial voluntarily.

\section{Strategies to improve adherence to interventions $\{11 \mathrm{c}\}$}

The number and time of taking medicine by every participant will be recorded. Similarly, unused drugs will be required to be returned and recorded during each visit. 


\section{Relevant concomitant care permitted or prohibited during the trial $\{11 d\}$}

Conventional rehabilitation and antiplatelet agents will be decided by physicians, and other TCM with the effect of nourishing Yin and promoting blood circulation is prohibited.

\section{Provisions for post-trial care $\{30\}$}

Not applicable, there is no prospective harm from this trial.

\section{Outcomes $\{12\}$}

\section{Primary outcome measures}

The primary measure of our study is the Modified Ashworth Scale (MAS), which to evaluate spasticity of hemiplegia limb at baseline, 1 month, 2 months, and 3 months after treated with Sangdantongluo granule. The MAS score ranges from 0 to 4 , and the higher score indicates more severe spasticity [11].

\section{Secondary outcome measures}

(1) Compopsite Spasticity Scale (CSS), to evaluate the spasticity of lower limb at baseline, 1 month, 2 months, and 3 months after treated with Sangdantongluo granule.

(2) Simplified Fugl-Meyer Motor Scale (S-FM) [12], to assess the movement at baseline, 1 month, 2 months, and 3 months after treated with Sangdantongluo granule.

(3) National Institute of Health stroke scale (NIHSS), to assess the neurological deficit at baseline, 1 month, 2 months, and 3 months after treated with Sangdantongluo granule.

(4) Modified Rankin Scale (mRS), to evaluate the neurofunctional recovery at baseline, 1 month, 2 months, and 3 months after treated with Sangdantongluo granule.

(5) Modified Barther Index (MBI), to evaluate the activities of daily living at baseline, 1 month, 2 months, and 3 months after treated with Sangdantongluo granule.

(6) Surface electromyography, to evaluate the spasticity of hemiplegia limb at baseline, 2 months after treated with Sangdantongluo granule.

\section{Participant timeline $\{13\}$}

Participant recruitment will begin in June 2021 and will end in May 2023. Time schedule of enrolment, interventions, assessments, and visits for participants are listed in Table 4.

\section{Sample size $\{14\}$}

According to the literature,"Meng Wang, Shouguo Liu, Zhihang Peng et al. Tibetan Medicated Bathing Therapy for Patients With Post-stroke Limb Spasticity: A Randomized Controlled Clinical Trial” [13]. After the combined treatment of Tibetan medicated bathing therapy and conventional rehabilitation for 3 months, MAS changes were $0.27 \pm 0.66$ in conventional rehabilitation group, and $0.65 \pm 0.75$ in Tibetan medicated bathing 
group. We believe that Sangdantongluo granule should increase the change value of MAS by 0.1 compared with the Tibetan medicine bathing studied by Wang et al. With $\alpha=0.025$ and test efficiency $1-\beta=0.8$, we will require at least 112 subjects. Considering the $15 \%$ abscission rate and the rounding principle, 132 participants in total (66 in each group) will be enrolled.

\section{Recruitment $\{15\}$}

Three strategies will be undertaking to guarantee adequate participant enrolment:

(1) posting recruitment advertisement in conspicuous places in hospital;

(2) publishing and disseminating recruitment ads through WeChat, a popular social software in China;

(3) introducing the clinical trial to PSS patients through the physician.

\section{Assignment of interventions: allocation}

\section{Sequence generation $\{16 a\}$}

By using the method of block randomization, the random number grouping table is generated with the process statement of the SAS statistical software Proc Plan, and then the random assignment scheme is formed. The random allocation scheme, the selected area length and random initial seed parameters were taken as blind bottom, sealed and hidden in an opaque envelope, and handed over to the unit in charge of the project for safekeeping. In this study, patients were included and drug numbers were issued strictly according to the order of random assignment table numbers. The online randomization system was used for the implementation of randomization. During the research process, researchers logged in the system to apply for the randomization codes assigned by the system in order.

\section{Concealment mechanism \{16b\}}

The random coding table will be generated by the central random platform and sealed by the GCP specialist in Hunan Academy of Chinese Medicine Affiliated Hospital, which will be submitted to the project leader for preservation. The drugs will be packaged and confirmed as a blinded treatment by a GCP specialist; the random codes will be mentioned on the drugs and the box label.

\section{Implementation $\{16 c\}$}

The allocation sequence will be generated by professor Linyong Xu's team, an eminent statistician of Central South University in China. Neither the researcher nor the participants know the random sequences.

\section{Assignment of interventions: Blinding}

\section{Who will be blinded $\{17 \mathrm{a}\}$}

ALL researcher, participants, and statistician will be blinded throughout the course of trial.

\section{Procedure for unblinding if needed $\{17 b\}$}

For emergency blinding, there will be an emergency envelope for every case, which is recorded the 
information about the coding and groups. It is necessary to check the drugs used by the patients in case of any serious event, and patients need to be treated. The investigator should report to the project leader before unblinding. The project leader decides to initiate unblinding and open the corresponding emergency envelope.

\section{Data collection and management}

\section{Plans for assessment and collection of outcomes $\{18 \mathrm{a}\}$}

In this 12-week trial, all participants will be treated with the Sangdantongluo granule or placebo for 8 weeks, with follow-up continuing for another 4 weeks. Independent assessors at each center, who were trained before enrollment, will be responsible for duplicate measurements including MAS, CSS, S-FM, NIHSS, mRS, $\mathrm{MBI}$, and standardized assessment of surface electromyography at 4 weeks, 8 weeks, and 12 weeks. Data will be recorded in the online electronic data collection system (EDC).

\section{Plans to promote participant retention and complete follow-up \{18b\}}

All investigators and doctors will be advised to treat the participants in a friendly and respectful way and encourage them to participate. All researchers also will be advised provide health consultation or preregistration of specialist outpatient. The patients' round-trip fare will be reimbursed, which encourage them to finish the follow-up. The researchers will send reminders to the patients 2 to 3 days before each follow-up.

\section{Data management $\{19\}$}

The electronic data collection system (EDC) will be used for on-line data acquisition in our clinical trial. In order to ensure the security and privacy of the original data, the research team will arrange full-time clinical research coordinator for data entry to avoid problems related to data transmission. The data management schedule will be arranged by the data administrator prior to the trial, which containing research objectives, general design, EDC system administrator, research man, data administrator, statistical analyst, data validation, database locking, and data security. After the completion of data management, the data management report will be written by the data administrator, and the statistical analysis will be conducted after the approval of the research institution, statistician and bid sponsor.

\section{Confidentiality $\{27\}$}

The name and identity card number of patients will not be recorded in the data collection. The ID of patients will be showed initials, and outpatient or inpatient number. The researchers should not discuss the patients' condition and information in public areas, and patient data cannot be released in the media or on the internet. All documents will be stored confidentially and will only be accessible to members of the trial team. The online EDC will be assigned specific permissions to the different groups of researchers in the project to ensure the security and confidentiality of data. All data collected in the system will be backed up with reliable data and privacy security mechanisms, and desensitization technology will be adopted to ensure absolute 
privacy of the data of project participants. The data will be made available to other researchers upon request after the analyses and publication of primary findings.

Plans for collection, laboratory evaluation and storage of biological specimens for genetic or molecular analysis in this trial/future use $\{33\}$

Not applicable.

\section{Statistical methods}

\section{Statistical methods for primary and secondary outcomes $\{20 \mathrm{a}\}$}

Statistical analysis will be carried out by a professional team, who blinded to the whole course of the trial. The qualitative data of the two groups will be compared and analyzed by using chi-squared test or Wilcoxon test. And the quantitative data in accordance with the normal distribution will be compared and analyzed by using Student's t tests, whereas, the quantitative data which does not comply with the normal distribution will be compared and analyzed by using Wilcoxon test. The two-sided test will be adopted in the testing of hypotheses, and the test statistics and their corresponding $P$ values will be offered. Two-side $P$ value of $\leq$ 0.05 is regarded as statistically significant, and $P \leq 0.01$ as highly statistically significant.

\section{Interim analyses $\{21 b\}$}

We will begin to conduct statistical analysis when the sample reaches 66 . Principal investigator will decide whether to continue the experiment, according to the interim results.

\section{Methods for additional analyses (e.g. subgroup analyses) \{20b\}}

We will make subgroup analysis based on hemorrhagic stroke or ischemic stroke.

\section{Methods in analysis to handle protocol non-adherence and any statistical methods to handle missing data $\{20 \mathrm{c}\}$}

Participants who discontinued will be excluded from statistical analysis, and missing data will be estimated by multiple imputation.

\section{Plans to give access to the full protocol, participant level-data and statistical code} $\{31 c\}$

The full protocol, participant level-data and statistical code will be authorized by the corresponding author. 


\section{Oversight and monitoring}

\section{Composition of the coordinating centre and trial steering committee $\{5 \mathrm{~d}\}$}

The coordinating center is set up in Neurology Department of Hunan Academy of Chinese Medicine Affiliated Hospital. The primary investigator is the director of the department, who in charge of identifying potential recruits, acquiring consent, and messaging to the trail steering committee. The rial steering committee is set up in Good Clinical Practice office of Hunan Academy of Chinese Medicine Affiliated Hospital, whose responsibilities including revise and agreement of the protocol, supervising trial, reviewing progress of the trial and the paper for publication.

\section{Composition of the data monitoring committee, its role and reporting structure $\{21 \mathrm{a}\}$} The data monitoring committee (DMC), who independent from the sponsor and free from competing interests, will ensure the interests of participants, the accurate of trial sheet and reported data, and follow of the registered protocol.

\section{Adverse event reporting and harms $\{22\}$}

Adverse events, including symptom, time, duration, interventions taken and outcome, will be recorded and presented to researchers and ethics committee, who will decide whether to discontinue the treatment.

\section{Frequency and plans for auditing trial conduct $\{23\}$}

The trial management team will meet every three months to review the study, and ethics committee will meet every six months to review the trial conduct.

Plans for communicating important protocol amendments to relevant parties (e.g. trial participants, ethical committees) $\{25\}$

Once there are significant changes to the protocol, we will inform the sponsor, ethics committees and subcenters.

\section{Dissemination plans $\{31 \mathrm{a}\}$}

The results will be published in peer-reviewed academic journals and shared with the general public and medical professionals.

\section{Discussion}

High cost on PSS brings out weightily economy burden for government, family and patients, due to the highly 
disability. Direct costs of PSS patients are fourfold as high in comparison with the stroke patients who do not have spasticity for the first year after stroke, which mainly because of the frequently hospitalization of PSS patients $[14,15]$. Consequently, effective spasticity management strategies are crucial to relieve the financial burden and elevate the quality of life of patient with PSS.

TCM is a generally accepted treatment with minimal side effects for PSS patients in China. There are increasing quantities of clinical studies on the treatment of PSS with TCM, such as orally Chinese herbal formulas, Chinese massage, acupuncture, medicated bath and so on [16-18]. The mechanism is related to modulation of gamma-aminobutyric acid and glutamate levels, reducing inflammatory response, and decreasing muscular tension [19-21]. Systematic review show that oral or topical application of TCM is beneficial to relieving spasticity of the upper and lower limbs in PSS patients. Whereas, the methodological quality of these clinical researches is quite low, lacking suitable means for random sequence generation, allocation concealment. Most importantly, only a few studies had used appropriate placebo to blind researchers or participants [8].

Sangdantongluo granule, a modern patent TCM, is mainly composed of Mori Fructus, Lycii Fructus, Salviae Miltiorrhizae Radix Et Rhizoma, and other 7 Chinese medicinal herbs. It is widely used in clinical practice to treat stroke, particularly PSS. Our previous research had confirmed that Sangdantongluo granule can relieve spasticity of hemiplegia limbs and improve motor performance in PSS patients. For the purpose of evaluating the clinical efficacy and safety of Sangdantongluo granule, we intend to conduct a multicenter, randomized, double-blind and placebo-controlled clinical study. We are aiming at providing high-quality evidence for Sangdantongluo granule in the treatment of PSS.

\section{Trial status}

The protocol version number is V 3.3 and the date is 09 Feb 2021. Participant recruitment will begin in July 2021 and will end in May 2023.

\section{Abbreviations}

PSS: Post-stroke spasticity; TCM: Traditional Chinese medicine; GCS:Glasgow Coma Scale; CSS:

Compopsite Spasticity; S-FM: Simplified Fugl-Meye; MAS: Modified Ashworth Scale; MBI: Modified Barther Index; mRS: Modified Rankin Scale; NIHSS: National Institute of Health Stroke; ALT: Alanine Aminotransferase; AST: Aspartate Aminotransferase; Cr: Creatinine; EDC: Electronic data collection system; DMC: Data monitoring committee

\section{Declarations}

Trials guidance: All manuscripts must contain the following subheadings:

- Acknowledgements

- Authors' contributions

- Funding 
- Availability of data and material

- Ethics approval and consent to participate

- Consent for publication

- Competing interests

- Authors' information (optional)

\section{Acknowledgements}

None.

\section{Authors' contributions $\{31 \mathrm{~b}\}$}

DW is the Chief Investigator. DW, LX, ZY, and YX designed the study. LX, YX, JJ, TY, RF, and YL will conduct the study. GM, RF and YX will analyze statistical data. All authors have read and agreed with the final manuscript.

\section{Funding $\{4\}$}

This study was supported by the Foundation of China Center for Evidence Based Traditional Chinese Medicine [grant number 2019XZZX-NB004].

\section{Availability of data and materials $\{29\}$}

All data will be provided by the corresponding author without any restriction.

\section{Ethics approval and consent to participate $\{24\}$}

The trial and protocol were approved by the Ethics Committee of Hunan Academy of Chinese Medicine Affiliated Hospital (No. [202102]20). The participants will be informed the trial protocols and consent before the trial begins, and their personal details will be kept confidential.

\section{Consent for publication $\{32\}$}

Not applicable.

\section{Competing interests $\{28\}$}

The authors declare that they have no competing interests. .

\section{Authors' information (optional)}

None. 


\section{References}

1. Wu SM, Wu B, Liu M, et al. Stroke in China: advances and challenges in epidemiology, prevention, and management. Lancet Neurology. 2019;18:394-405.

2. Zhou MG, Wang HD, Zeng XY, et al. Mortality, morbidity, and risk factors in China and its provinces, 1990-2017: a systematic analysis for the Global Burden of Disease Study 2017. Lancet. 2019;394:1145-58.

3. Zeng HL, Chen J, Guo Y, et al. Prevalence and Risk Factors for Spasticity After Stroke: A Systematic Review and Meta-Analysis. Frontiers in Neurology. 2021;11.

4. Schinwelski MJ, Sitek EJ, Waz P, et al. Prevalence and predictors of post-stroke spasticity and its impact on daily living and quality of life. Neurologia I Neurochirurgia Polska. 2019;53:449-57.

5. Kuo CL, Hu GC. Post-stroke Spasticity: A Review of Epidemiology, Pathophysiology, and Treatments. International Journal of Gerontology. 2018;12:280-4.

6. Cha YS, Arami A. Quantitative Modeling of Spasticity for Clinical Assessment, Treatment and Rehabilitation. Sensors. 2020;20.

7. Gupta AD, Chu WH, Howell S, et al. A systematic review: efficacy of botulinum toxin in walking and quality of life in post-stroke lower limb spasticity. Systematic Reviews. 2018;7.

8. Cai YY, Zhang CS, Liu SN, et al. Add-On Effects of Chinese Herbal Medicine for Post-Stroke Spasticity: A Systematic Review and Meta-Analysis. Frontiers in Pharmacology. 2019;10.

9. Association NSoCM. Chinese Guidelines for the Prevention of Stroke in Patients with Stroke and Transient Ischemic Attack 2014. Chinese Journal of Neurology. 2015;48:258-71.

10. Association NSoCM. Chinese Guidelines for early rehabilitation of stroke. Chinese Journal of Neurology. 2017;50:405-12.

11. Meseguer-Henarejos AB, Sanchez-Meca J, Lopez-Pina JA, et al. Inter- and intra-rater reliability of the Modified Ashworth Scale: a systematic review and meta-analysis. European Journal of Physical and Rehabilitation Medicine. 2018;54:576-90.

12. Hsueh IP, Hsu MJ, Sheu CF, et al. Psychometric Comparisons of 2 Versions of the Fugl-Meyer Motor Scale and 2 Versions of the Stroke Rehabilitation Assessment of Movement. Neurorehabilitation and Neural Repair. 2008;22:737-44.

13. Wang M, Liu SG, Peng ZH, et al. Tibetan Medicated Bathing Therapy for Patients With Post-stroke Limb Spasticity: A Randomized Controlled Clinical Trial. Journal of the American Medical Directors Association. 2020;21:374-+.

14. Lundstrom E, Smits A, Borg J, et al. Four-Fold Increase in Direct Costs of Stroke Survivors With Spasticity Compared With Stroke Survivors Without Spasticity The First Year After the Event. Stroke. 2010;41:319-24. 
15. Rychlik R, Kreimendahl F, Schnur N, et al. Quality of life and costs of spasticity treatment in German stroke patients. Health Economics Review. 2016;6.

16. Wang M, Liu SG, Peng ZH, et al. Effect of Tui Na on upper limb spasticity after stroke: a randomized clinical trial. Annals of Clinical and Translational Neurology. 2019;6:778-87.

17. Li HQ, Long DH, Li B, et al. A clinical study to assess the influence of acupuncture at "Wang's Jiaji" acupoints on limb spasticity in patients in convalescent stage of ischemic stroke: study protocol for a randomized controlled trial. Trials. 2019;20.

18. Qiu X, Gao YC, Zhang ZX, et al. Fire Acupuncture versus conventional acupuncture to treat spasticity after stroke: A systematic review and meta-analysis. Plos One. 2021;16.

19. Huang J, Tao J, Xue XH, et al. Gua Lou Gui Zhi decoction exerts neuroprotective effects on post-stroke spasticity via the modulation of glutamate levels and AMPA receptor expression. International Journal of Molecular Medicine. 2013;31:841-8.

20. Hu HX, Li ZF, Zhu XQ, et al. Gua Lou Gui Zhi decoction suppresses LPS-induced activation of the TLR4/NF-kappa B pathway in BV-2 murine microglial cells. International Journal of Molecular Medicine. 2013;31:1327-32.

21. Zhu WZ, Zheng GQ, Gu Y, et al. Clinical efficacy and sEMG analysis of a new traditional Chinese medicine therapy in the treatment of spasticity following apoplectic hemiparalysis. Acta Neurologica Belgica. 2014;114:125-9. 
Figures

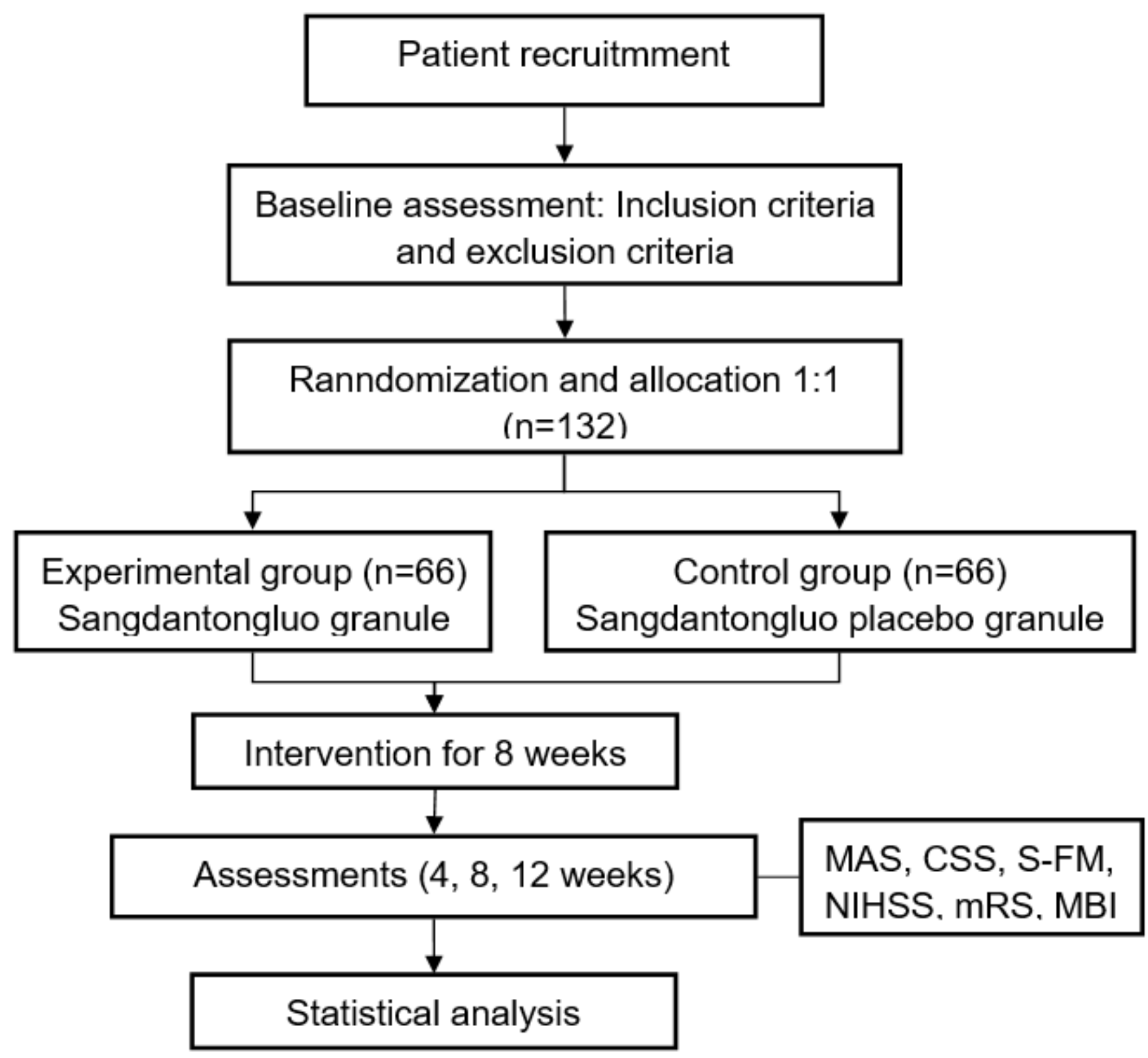

Figure 1

Flow diagram of the trial process 